\title{
OBJECT-BASED CHANGE DETECTION FOR INDIVIDUAL BUILDINGS IN SAR IMAGES CAPTURED WITH DIFFERENT INCIDENCE ANGLES
}

\author{
Junyi Tao ${ }^{1}$, Stefan Auer ${ }^{2}$, Peter Reinartz ${ }^{1}$, Richard Bamler ${ }^{1,2}$ \\ ${ }^{1}$ Remote Sensing Technology Institute, German Aerospace Center (DLR), Oberpfaffenhofen, Germany \\ ${ }^{2}$ Chair of Remote Sensing Technology, Technische Universität München (TUM), Munich, Germany
}

\begin{abstract}
Change detection of two SAR images captured with different incidence angles is a difficult task but may be important in urgent situations like earthquakes. This paper presents a simulation based algorithm to detect negative changes of buildings in two high resolution SAR images captured with different incidence angles. The analysis is supported by LiDAR data where individual wall models are extracted and are simulated to predict their shape in the SAR images. Afterwards, point signatures within the layover areas are extracted, converted to the same geometry, and are compared with a buffer change detection algorithm. The proposed method is tested for several buildings (in Munich city center) imaged in TerraSAR-X spotlight mode.
\end{abstract}

Index Terms - SAR simulation, wall extraction, change detection, LiDAR, TerraSAR-X

\section{INTRODUCTION}

High resolution synthetic aperture radar (SAR) images have been exploited for different change detection applications, like damage assessment [1], surveillance [2], and have shown great potentials. These applications are based on the comparison of pre- and post-event space borne SAR images captured with the same signal incidence angle. However, because of the satellite orbit trajectory - e.g. for TerraSAR$\mathrm{X}$ the maximum site access time is 2.5 days (adjacent orbit) and the revisit time is 11 days (same orbit) - the first available post-event SAR image may be captured with a different incidence angle. In urgent situations such as earthquakes, this data has to be analyzed for changes in order to support local decision makers as fast as possible.

As presented in Fig. 3, the same building appears differently in SAR images captured with different signal incidence angles: i) wall layover areas are scaled in range direction, ii) object occlusions are different, affecting the object visibility, shadow size, etc. iii) multiple reflections of signals related to building structures may be different. Accordingly, a pixel based comparison is not suitable as it may lead to a large amount of false alarms.
In this paper, a simulation method is applied in order to predict the geometric shape of building facades in TerraSAR-X images captured with different imaging geometries. A digital surface model (DSM) based on LiDAR data is included in order to provide a-priori knowledge about the building shape in the SAR images. Based on the simulation results, the layover areas corresponding to individual facades in the SAR images are extracted and compared. To this end, point signatures within the layover areas, are extracted and projected to the same geometry. Thereafter, their positions are compared with a buffer change detection algorithm following the idea of [6].

\section{METHODOLOGY}

The underlying scenario is that a DSM and two SAR data are captured at times $t_{0}, t_{1}$ and $t_{2}$, respectively. The aim is to detect negative changes (e.g. collapsed or removed buildings) between time points $t_{1}$ and $t_{2}$. In this context, the basic idea is to focus on changes of facade layover areas in SAR images. For predicting the facade layover areas, individual wall models are extracted from a LiDAR DSM (section 2.1). The geometric relationship of the corresponding layover areas in SAR images with different signal incidence angles is discussed in section 2.2. At last, point features from the wall layover areas are extracted, converted to the same geometry and are compared (section 2.3).

\subsection{Extraction of wall models from a building model}

From a given DSM, a normalized digital surface model (nDSM) can be generated [3]. Isolated parts in the nDSM exceeding a size threshold (e.g. > 1000 pixels) are selected as buildings of interest. The method for decomposing individual building model into separate wall segments is as follows:

1. Gradient magnitude and gradient direction maps are generated and are processed with a median filter.

2. A height threshold value is calculated in the neighborhood $(3 \times 3$ patch) of the pixel with the highest gradient magnitude, which equals the mean value of the maximal and minimal height in the patch. 
3. After extracting all height values above the height threshold, the building boundary polygons (with 1 pixel width) are generated. These boundary polygons may describe building outside walls, courtyards, and even walls of different building blocks.

4. For every boundary polygon, the corresponding gradient direction values are extracted. A strong variation of the gradient direction along the polygon indicates a corner of the building. The boundary polygon is then separated at these corner pixels into boundary segments.

5. The boundary segments with 1 pixel width are enlarged using the pixel neighborhood (dilation of 3 pixels) and similar gradient direction (difference smaller than $30^{\circ}$ ), resulting in wall masks for the building facades.

6. The wall masks are fused into a wall map as output, in which pixels of one wall model share the same integer value. Besides, different wall parameters are generated which are useful for choosing walls of interest (median gradient direction, length, height) and for wall layover conversion (median gradient direction, position of the wall center point).

Due to the height threshold in the second step, boundaries of low building parts will not be detected in our approach. However, this limitation is not major as facades with low height will trigger only a low number of signatures in the SAR images. The suggested threshold value for strong variations of the gradient direction in step 4 is 30 degrees, which works for most of the rectangular buildings.

\subsection{Wall layover conversion}

Using the separated wall models as input for an automatic processing chain of simulation [4] based on RaySAR [5], simulated images of the wall models are generated. These geocoded images can be directly compared to the TerraSAR-X GEC products [4]. The simulated wall layover images are converted to binary masks and the corresponding SAR image sections are extracted.

The correspondence of image pixels in wall layover areas and the building façade can be found using the geoinformation of the wall model (the position and gradient direction of the wall) and the projection geometry of SAR GEC product. Based on this geometric relationship, the extracted wall layover parts of different SAR images are converted to the same image geometry. This principle is illustrated in Fig.1.

The building wall is assumed to be a vertical plane, which can be described with one point (wall center) and one direction (wall median gradient direction), provided by the wall extraction step in section 2.1. Given azimuth direction and the signal incidence angle, a point $\mathrm{P}_{1}$ in the layover area of SAR image 1 can be projected to this vertical plane with an intersection point $\mathrm{P}_{0}$. Considering the imaging geometry of SAR image 2 with another azimuth and signal incidence angle, this point $\mathrm{P}_{0}$ is then projected to the horizontal plane of the SAR GEC image 2.

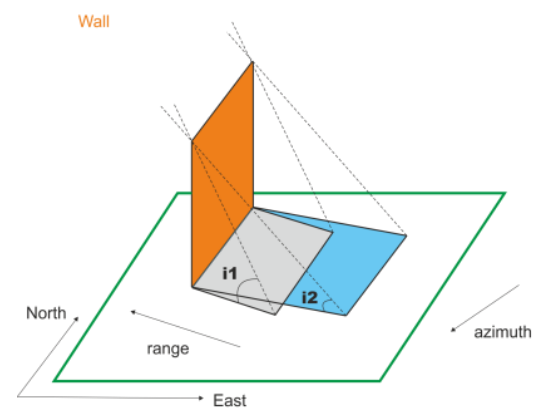

Figure 1. The principle of wall layover conversion.

It is worth noting that the assumption of a planar wall leads to errors in the conversion. In reality, not all facade structures related to point signatures will be arranged in a plane. Accordingly, the error of the converted point position is proportional to the distance between the real point and the assumed vertical plane.

\subsection{Buffer change detection using point features}

The previous steps provide wall layover images for different imaging geometries. For comparing them a buffer change detection method is used which is based on [6]. This method was originally developed for geoinformation system (GIS) applications, e.g., for comparing road maps. In our case, point features (local maximum points within the wall layover) are compared. Thereto, the processing follows four main steps:

1. The local maximum points of the two SAR wall layovers are extracted. The points with low intensity (e.g. < 700) are less important and are discarded.

2. The extracted point features from SAR image 1 are converted to the $2^{\text {nd }}$ SAR imaging geometry using the method described in 2.2.

3. For every converted point of SAR image 1, its Euclidean distances to all point features of SAR image 2 are calculated. In case one distance is smaller than a pre-defined buffer distance, the point is considered as being "inside". Otherwise, it is an "outside" point.

4. The third step is conducted for all converted points (with total point number of $L_{\text {total }}$ ). The number of "inside" points is $L_{\text {inside }}$. The change ratio is then defined as:

$$
P_{\text {point }}=1-\frac{L_{\text {inside }}}{L_{\text {total }}}
$$

The buffer distance used in the third step mainly depends on the accuracy of the wall center point coordinate, the wall gradient direction, the assumption of planar facades and the accuracy of the extracted local maximum points. Furthermore, Using the procedure described in section 2.2, the propagation of uncertainty of the converted point 
positions depending on the input parameters (wall direction, wall center point position, position of extracted local maximum points) are calculated. Based on this, the buffer distance for every wall model is calculated, resulting in buffer values between 2 and 6 pixels.

\section{EXPERIMENTAL RESULTS}

To assess the potential of the proposed approach, experiments were carried out on a LiDAR DSM and two TerraSAR-X GEC products. The LiDAR data (Fig. 2) is derived at April 2003 for Munich city centre with vertical and horizontal resolution of 0.1 meter and 1 meter, respectively. The two SAR images (Fig. 3) have been acquired from descending orbits with incidence angles of $25.3^{\circ}$ and $39.3^{\circ}$ on May $26^{\text {th }} 2008$ and January $5^{\text {th }} 2010$, respectively.

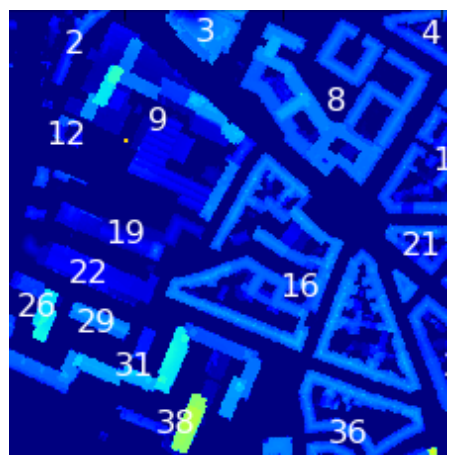

Figure 2. Extract of the used DSM with building IDs.

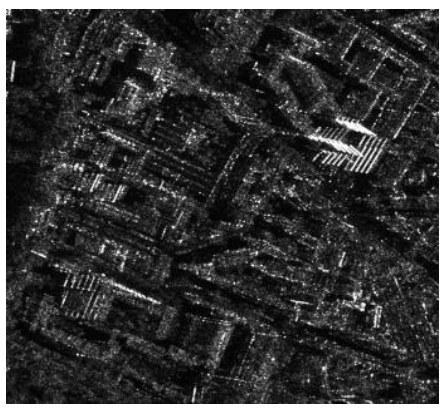

(a) 2008-05-26, $25^{\circ}$

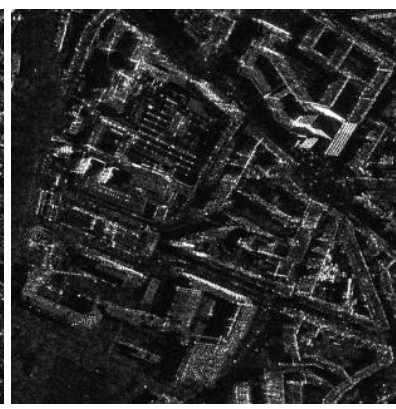

(b) $2010-01-05,39^{\circ}$
Figure 3. TerraSAR-X amplitude images of Munich test site, located in east and north direction.

From the DSM (Fig. 2), individual building models are extracted. Their IDs are visualized at the building center in the DSM. As an example, building 26 is selected for detecting changes and is shown in Fig. 4a. Using the method described in section 2.1, individual wall models are extracted from this building model. Fig.4b shows the wall masks with their wall IDs.

Additionally, the wall parameters are calculated (see Table 1). As the azimuth angles of the two input SAR images are approx. $188^{\circ}$, only the walls $1-5$ are "visible" to the SAR sensor. The walls 1, 2, and 4 have aspect angles bigger than $50^{\circ}$ relative to the sensor. Their corresponding signatures are hardly separable as the layover areas are very narrow in azimuth. Only the walls 3 and 5 (named 26_3 and 26_5) are of relevant size (e.g. length and height $>10 \mathrm{~m}$ ) and are chosen for the layover change detection.

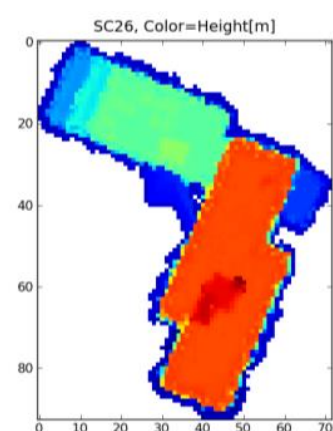

(a)

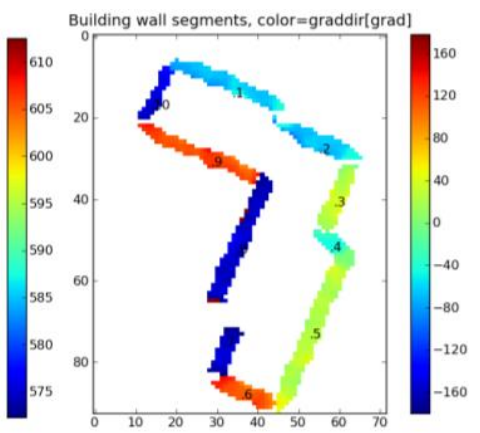

(b)
Figure 4. Wall extraction from the building model 26. (a) building model, color indicates height (b) extracted wall masks with wall IDs, color indicates gradient direction.

TABLE 1. Wall parameters of building 26

\begin{tabular}{|c|c|c|c|}
\hline wall id & $\begin{array}{c}\text { gradient direction } \\
\text { [degrees] }\end{array}$ & wall length [m] & height [m] \\
\hline 1 & $-64,6$ & 29,5 & 18,4 \\
\hline 2 & $-64,9$ & 20,1 & 28,9 \\
\hline 3 & 24,1 & 18,4 & 26,7 \\
\hline 4 & $-44,1$ & 7,1 & 19,4 \\
\hline 5 & 24,9 & 40,7 & 31,3 \\
\hline 6 & 112,9 & 15,7 & 31,3 \\
\hline 7 & $-152,5$ & 17 & 26,5 \\
\hline 8 & $-157,1$ & 32,6 & 26,6 \\
\hline 9 & 115,2 & 30,4 & 18,3 \\
\hline 10 & $-145,9$ & 14,3 & 18,3 \\
\hline
\end{tabular}

The corresponding part of the chosen wall mask in the DSM is extracted as a wall model. This wall model is simulated and the resulting layover image is geocoded. Thereafter, the wall layover is converted to a mask and the corresponding real SAR image is extracted. Fig. 5 shows the layover of the wall 26_5 in two SAR images. The red points indicate the extracted local maximum points from the SAR image. The cyan circles in Fig. 5b indicate the projected positions of the red points from Fig. 5a. Only a few converted points (cyan) in Fig. $5 \mathrm{~b}$ correspond to a near red point. The change ratio for the wall 26_5 is 0.889 , which means $88.9 \%$ of the converted points are "outside" (see the second row in Table 2 ). This result indicates that this wall is negative changed. Compared to this detected negative change, Fig. 6 shows an unchanged wall 8_20 of building 8. As it is shown in Fig. $6 \mathrm{~b}$, most of the converted points (cyan) have a red point nearby, indicating that they are still present (see low change ratio in $6^{\text {th }}$ row in Table 2). However, some points (e.g. at the right side of Fig. 6a) are "outside", as they are signatures related to an adjacent wall. 


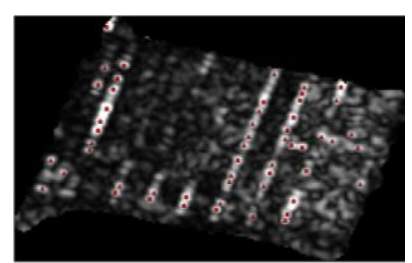

(a) $2008-05-26,25^{\circ}$

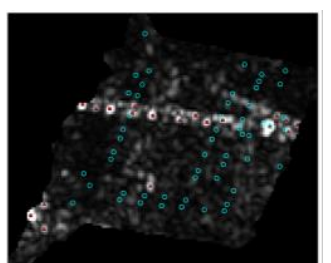

(b) 2010-01-05, 39

Figure 5. SAR images with extracted (red) and converted (cyan) local maximum points of wall 265 .

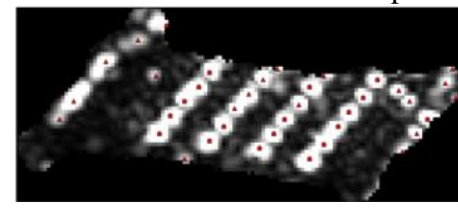

(a) $2008-05-26,25^{\circ}$

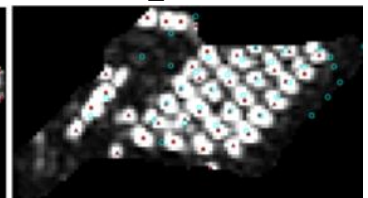

(b) 2010-01-05, 39
Figure 6. SAR images with (red) and converted (cyan) local maximum points of wall 8_20.

Table 2 summarizes the buffer change detection results of several selected walls. An ideal change ratio for unchanged building should be zero. Most of the unchanged buildings in this table have a change ratio varies from 0.2 to 0.6 . The main reason is that the algorithm has only considered the main impact of different incidence angles - the scaling of wall layover. In contrast, the other effects (e.g. different occlusions, mixture of signatures from other objects) are not covered, leading to "outside" points for every tested building.

For a final change decision, the suggested decision rule of the change ratio may be: change, $0.8-1.0$; unchanged, $0-0.6$; change candidate 0.6-0.8. Additional change detection parameters may help to further categorize the change candidates. Based on this rule, the first two walls in Table 2 are changed, what corresponds to reality.

TABLE 2. Buffer change detection results of several selected walls

\begin{tabular}{|c|c|c|c|}
\hline Wall ID & $\begin{array}{c}\text { Number of extracted } \\
\text { Points from SAR 1 }\end{array}$ & $\begin{array}{c}\text { Number of extracted } \\
\text { Points from SAR 2 }\end{array}$ & change ratio \\
\hline $26 \_3$ & 45 & 4 & 0,933 \\
\hline $26 \_5$ & 54 & 19 & 0,889 \\
\hline $38 \_1$ & 10 & 15 & 0,500 \\
\hline $38 \_2$ & 49 & 100 & 0,776 \\
\hline $8 \_18$ & 100 & 100 & 0,330 \\
\hline $8 \_20$ & 38 & 39 & 0,395 \\
\hline $31 \_14$ & 26 & 47 & 0,500 \\
\hline $31 \_13$ & 25 & 63 & 0,520 \\
\hline $29 \_1$ & 46 & 51 & 0,217 \\
\hline $29 \_2$ & 11 & 18 & 0,636 \\
\hline $16 \_19$ & 27 & 29 & 0,519 \\
\hline $16 \_34$ & 34 & 32 & 0,588 \\
\hline
\end{tabular}

\section{CONCLUSION}

This paper presents a method for detecting changes between two high resolution TerraSAR-X images captured with different signal incidence angles. Individual building wall models are extracted from a LiDAR DSM and are used to predict their shape in real SAR images with the support of a SAR simulator (RaySAR). The two SAR image sections corresponding to the same building façade are extracted. Thereafter, the detection of changes is based on the comparison (buffer change detection) of local maximum points which are converted to the same layover geometry

The proposed algorithm is applicable for identifying negative changes within the first available SAR dataset after an unexpected event, what may support urgent situations, e.g., the assessment of building damages.

In this context, several limitations are of relevance. First, the method is based on point features in wall layover areas which might be influenced by signatures coming from roofs, grounds or other adjacent objects. Moreover, the difference of incidence angles may lead to disappearance of the points because of the loss of visibility, possibly resulting in false alarms in our results. Finally, any changes between the acquisition time of LiDAR and the first SAR image are not considered in our approach.

Nonetheless, the proposed method enables to give hints on significant building changes and offers first attempts in detecting changes of facades despite of a variation of the imaging geometry. So far, the method has been tested for different building blocks in Munich and shows promising results. Future work will concentrate on extending the methodology by elaborating further complementary change detection parameters.

\section{ACKNOWLEDGMENT}

The TerraSAR-X data were provided by DLR through the Science Projects MTH0505. The LIDAR data is provided by Landesamt für Vermessung und Geoinformation Bayern.

\section{REFERENCES}

[1] F. Bovolo and L. Bruzzone, "A split-based approach to unsupervised change detection in large-size multitemporal images: Application to tsunami-damage assessment," IEEE Trans. Geosci. Remote Sens., vol. 45, no. 6, pp. 1658 -1670, 2007.

[2] F. Bovolo, C. Martin, L. Bruzzone, "A Novel Hierarchical Approach to Change Detection with Very High Resolution SAR Images for Surveillance Applications," IGARSS 2012, Munich.

[3] H. Arefi, P. d'Angelo, H. Mayer, and P. Reinartz, "Iterative approach for efficient digital terrain model production from CARTOSAT-1 stereo images," J. Appl. Remote Sens., 5(1), 2011.

[4] J. Tao, S. Auer, and P. Reinartz, "Detecting changes between a DSM and a high resolution SAR image with the support of simulation based separation of urban scenes," Proceedings of EUSAR 2012, Nuremberg, Germany, pp. 95-98, 2012.

[5] S. Auer, 3D Synthetic Aperture Radar Simulation for Interpreting Complex Urban Reflection Scenarios, Ph.D. thesis, Technische Universität München, 2011.

[6] H.G. Sui, Automatic change detection for road-networks base on features, Ph.D. thesis, Wuhan University, 2002 